\title{
A Simple Hybrid Synchronization for a Class of Chaotic Financial Systems
}

\author{
Jiming Zheng, Xiaoshuang Li, and Yang Qiu \\ School of Science, Chongqing University of Posts and Telecommunications, Chongqing 400065, China \\ Correspondence should be addressed to Jiming Zheng; zhengjm0903@qq.com
}

Received 19 June 2017; Revised 25 August 2017; Accepted 12 September 2017; Published 18 October 2017

Academic Editor: Francisco R. Villatoro

Copyright (C) 2017 Jiming Zheng et al. This is an open access article distributed under the Creative Commons Attribution License, which permits unrestricted use, distribution, and reproduction in any medium, provided the original work is properly cited.

\begin{abstract}
It is an important to achieve the hybrid synchronization of the chaotic financial system. Chaos synchronization is equivalent to the error system which is asymptotically stable. The hybrid synchronization for a class of finance chaotic systems is discussed. First, a simple single variable controller is obtained to synchronize two identical chaotic financial systems with different initial conditions. Second, a novel algorithm is proposed to determine the variables of the master system that should antisynchronize with corresponding variables of the slave system and use this algorithm to determine the corresponding variables in the chaotic financial systems. The hybrid synchronization of the chaotic financial systems is realized by a simple controller. At the same time, different controllers can implement the chaotic financial system hybrid synchronization. In comparison with the existing results, the obtained controllers in this paper are simpler than those of the existing results. Finally, numerical simulations show the effectiveness of the proposed results.
\end{abstract}

\section{Introduction}

Since the 1960s, with the discovery of chaotic systems, chaos has set off a nonlinear dynamic research boom $[1,2]$. The chaos phenomena in economics were first discovered in 1985 [3]. The chaos in the economic system meant that the macroeconomic movement itself had inherent instability. For the control of chaotic systems, the first study was carried out in [4], and they studied with a more systematic and strict parameter perturbation method, that is, the OGY method. The chaotic financial system was proposed in 1993 [5]. In 2004, a simplified financial system model was put forward by choosing an appropriate coordinate system and setting the appropriate dimensions for each state variable [6]. From then on, some scholars have done a lot of work on chaotic financial systems. However, there are still some limitations in the control and synchronization results. As far as we know, hybrid synchronization in the study of chaotic financial systems is poorly understood. Therefore, the chaotic financial systems need further discussion.

In 1990, Pecora and Carroll made chaos synchronization come true [7]. Chaotic synchronization control has become a favorite topic in nonlinear science and has been widely used in secure communications, automatic control, neural networks, and so on. Chaos synchronization of finance system has become a hot issue in the research and application of current chaos theory, and it is also a topical issue in the study of economic theory and method. Scholars have also done a lot of related work in the financial system, as well as in the control and synchronization research [8-10]. So far, a lot of methods about chaotic synchronization have been presented to prove that the chaotic synchronization method feasibly [11-15], such as driving response synchronization, coupled synchronization, feedback synchronization, impulsive synchronization, and adaptive synchronization, for the study of chaos synchronization problem has been more mature. However, compared with the chaotic synchronization problem, the problem of antisynchronization of chaotic systems is poorly known, so is the hybrid synchronization, and there are few studies in the financial system. In a broad sense, the synchronization and antisynchronization of chaotic systems are the special cases of the hybrid synchronization of the chaotic financial systems; that is, all the variables are synchronized or are antisynchronous [16]. 
In recent years, some scholars have obtained some research results in the antisynchronization of [12-15], but they are still in the initial stage; many theories are not mature yet. For example, in the study of antisynchronization problems, the equilibrium point of the error system $\dot{E}=F(y)+F(x)$ is not explicitly stated, if and only if the condition of $F(-x)=$ $-F(x)$ is satisfied $[13,14]$, so that the study of the antisynchronization has a lot of research prospects. And so far, in the study of chaos hybrid synchronization problem, there has not been any result of the explicit conditions or algorithms to choose which variables in the driving chaotic system can be synchronized or antisynchronized with the implementation of the corresponding variables in the response chaotic system. Scholars have made some achievements in the chaotic system in hybrid synchronization $[17,18]$, but the hybrid synchronization of the chaotic financial systems is still not perfect so far [15-17]. There is still a long wall to go.

In this paper, we discussed the synchronization and the hybrid synchronization of a class of chaotic financial systems. Under different initial conditions, two identical chaotic financial systems can achieve synchronization by designing a simple single variable controller, provide a criterion for variable chaotic system which can achieve antisynchronization algorithm, and applied it to this type of chaotic financial systems to achieve hybrid synchronization of chaotic financial systems, while the hybrid synchronization of the chaotic financial systems is realized by a simple controller. At the same time, in dealing with different situations, the controller can be changed, and the optimal controller can be chosen according to the length of the reaction time, so that the chaotic system can achieve synchronization and antisynchronization coexistence. In comparison with the existing results, the proposed controllers are simpler than those of the existing results. Finally, numerical simulations verify the correctness and the effectiveness of the proposed results.

\section{Synchronization of Chaotic Financial Systems by a Single Variable Controller}

The chaotic financial systems can be described by the following differential equation system: [5]:

$$
\begin{aligned}
& \dot{x}=z+(y-a) x \\
& \dot{y}=1-b y-x^{2} \\
& \dot{z}=-x-c z,
\end{aligned}
$$

where $x, y$, and $z$ represent the interest rate, investment demand, and price index, respectively; $a, b$, and $c$ are system parameters. And they are positive constants. By selecting an appropriate coordinate system and setting appropriate dimensions for each state variable, system (1) is transformed into simplified chaotic financial systems [6]:

$$
\begin{aligned}
& \dot{x}_{1}=F_{1}(x)=-m\left(x_{1}+x_{2}\right) \\
& \dot{x}_{2}=F_{2}(x)=-x_{2}-m x_{1} x_{3} \\
& \dot{x}_{3}=F_{3}(x)=n+m x_{1} x_{2},
\end{aligned}
$$

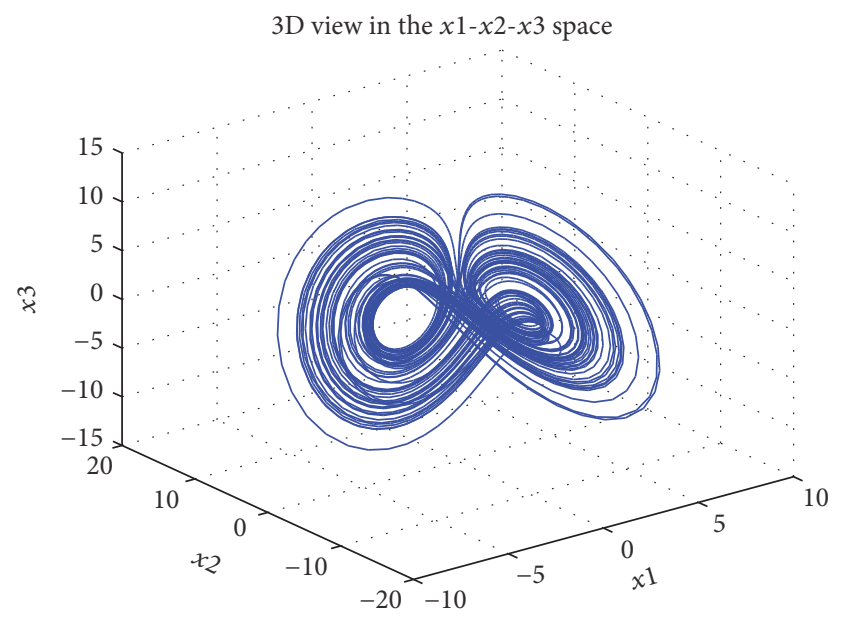

FIgURE 1: The chaotic attractor of the financial system (2).

where $F(x)=\left(F_{1}(x), F_{2}(x), F_{3}(x)\right)^{T}$ is a continuous vectorvalued function, $m, n$ are the system parameters, and $m, n>$ 0 . Let the right side of system (2) be zero; we can get two equilibrium points of system (2) as follows:

$$
\begin{aligned}
& p_{1}=\left(\frac{\sqrt{m n}}{n},-\frac{\sqrt{m n}}{n}, \frac{1}{m}\right), \\
& p_{2}=\left(-\frac{\sqrt{m n}}{n}, \frac{\sqrt{m n}}{n}, \frac{1}{m}\right) .
\end{aligned}
$$

If $m>1$, the two equilibrium points $p_{1}\left(p_{2}\right)$ of system (2) are unstable. In particular, when $m=3$ and $n=15$, starting with $x_{0}=(-2,3,4)^{T}$, system (2) is structurally unstable. Figure 1 shows the chaos and strange attractor of financial system (2).

In order to study the synchronization problem of the chaotic financial systems, let the system (2) be the driver system, and then the corresponding response system is as follows:

$$
\begin{aligned}
& \dot{y}_{1}=F_{1}(y)=-m\left(y_{1}+y_{2}\right) \\
& \dot{y}_{2}=F_{2}(y)=-y_{2}-m y_{1} y_{3} \\
& \dot{y}_{3}=F_{3}(y)=n+m y_{1} y_{2},
\end{aligned}
$$

where $F(y)=\left(F_{1}(y), F_{2}(y), F_{3}(y)\right)^{T}$ is also a continuous vector-valued function.

For systems (2) and (4), we give definitions of complete synchronization and antisynchronization [19] as follows.

Definition 1. Consider drive system (2) and response system (4). Suppose that there is a diagonal matrix $\Lambda=$ $\operatorname{diag}\left(\lambda_{1}, \lambda_{2}, \ldots, \lambda_{n}\right)$ that makes $\lim _{t \rightarrow+\infty}\|y-\Lambda x\|=0$ or $\lim _{t \rightarrow+\infty}\left|y_{i}-\lambda_{i} x_{i}\right|=0(i=1,2, \ldots, n)$. If $\lambda_{i}=1(i=$ $1,2, \ldots, n)$, then system (2) is called complete synchronization with system (4). If $\lambda_{i}=-1(i=1,2, \ldots, n)$, system (2) is called antisynchronization with system (4).

Let $e=\left(e_{1}, e_{2}, e_{3}\right)^{T}$ be the error space variable. The synchronization error is denoted by $e=y-x$. Hence 
the synchronization error system of drive system (2) and response system (4) can be expressed as follows:

$$
\dot{e}=F(y)-F(x) \text {. }
$$

So the error system is described as

$$
\begin{aligned}
& \dot{e}_{1}=F_{1}(x, e)=-m\left(e_{1}+e_{2}\right) \\
& \dot{e}_{2}=F_{2}(x, e)=-e_{2}-m\left(e_{1} e_{3}+x_{3} e_{1}+x_{1} e_{3}\right) \\
& \dot{e}_{3}=F_{3}(x, e)=m\left(e_{1} e_{2}+x_{1} e_{2}+x_{2} e_{1}\right) .
\end{aligned}
$$

From Definition 1, it is obvious that drive system (2) is fully synchronized with response system (4) if and only if $\lim _{t \rightarrow \infty}\|e(t)\|=0$. In order to discuss the synchronization control problem of error system (6), some assumptions are introduced firstly.

$\left(\mathrm{H}_{1}\right)$ If system (2) is a chaotic system and $F_{i}$ satisfies the uniform Lipschitz condition, then there is $\mu_{i}>0(i=1,2,3)$, which makes system (6) satisfy

$$
e_{i} F_{i}(x, e) \geq \mu_{i} e_{i}^{2} \quad(i=1,2,3) .
$$

$\left(\mathrm{H}_{2}\right)$ Consider the uncontrolled synchronization error system (6). If some $e_{i}=0(i=1,2,3)$, then the subsystem $\dot{e}_{j}=F_{j}(y)-F_{j}(x)=F_{j}\left(x, 0, e_{j}\right)(j=1,2,3 ; j \neq i)$ is asymptotically stable, the design of a single variable controller to achieve synchronization control.

$\left(\mathrm{H}_{3}\right)$ The design of a single variable controller is suited to $u_{i}=k_{1} e_{i}, u_{j}=0(i, j=1,2,3 ; j \neq i)$, where $k_{1}$ satisfies $\dot{k}_{1}=-\gamma e_{i}^{2}(i=1,2,3), \gamma$ is an arbitrary positive numbers and generally $\gamma=1$.

According to the control theory, synchronization error system (6) is represented by the controller $U_{1}=\left(u_{1}, u_{2}, u_{3}\right)^{T}$ as follows:

$$
\begin{aligned}
& \dot{e}_{1}=-m\left(e_{1}+e_{2}\right)+u_{1} \\
& \dot{e}_{2}=-e_{2}-m\left(e_{1} e_{3}+x_{3} e_{1}+x_{1} e_{3}\right)+u_{2} \\
& \dot{e}_{3}=m\left(e_{1} e_{2}+x_{1} e_{2}+x_{2} e_{1}\right)+u_{3} .
\end{aligned}
$$

In particular, if $e_{1}=0$ and $U_{1}=(0,0,0)^{T}$, then system (6) can obtain the following subsystems:

$$
\begin{aligned}
& \dot{e}_{2}=-e_{2}-m x_{1} e_{3} \\
& \dot{e}_{3}=m x_{1} e_{2} .
\end{aligned}
$$

System (9) is globally asymptotically stable at the origin.

In order to achieve system synchronization, we design the controller $U_{1}$ for $U_{1}=\left(k_{1} e_{1}, 0,0\right)^{T}$. Then control error system (6) can be expressed as

$$
\begin{aligned}
& \dot{e}_{1}=-m\left(e_{1}+e_{2}\right)+k_{1} e_{1} \\
& \dot{e}_{2}=-e_{2}-m\left(e_{1} e_{3}+x_{3} e_{1}+x_{1} e_{3}\right) \\
& \dot{e}_{3}=m\left(e_{1} e_{2}+x_{1} e_{2}+x_{2} e_{1}\right),
\end{aligned}
$$

where the feedback gain $k_{1}$ is adapted according to the following update law:

$$
\dot{k}_{1}=-\gamma e_{1}^{2} \text {. }
$$

Normally, make $\gamma=1$. System (10) and (11) can be called the augment system $(*)$ and introduce a Lyapunov function as follows:

$$
V_{1}\left(e, k_{1}\right)=\frac{1}{2} e^{T} e+\frac{1}{2 \gamma}\left(k_{1}+L_{1}\right)^{2}
$$

where

$$
L_{1}>M_{1} \sup _{x_{1} \neq 0} \frac{e_{2}^{2}+e_{3}^{2}}{e_{1}^{2}}, \quad M_{1}=\max _{1 \leq i \leq 3} \mu_{i} .
$$

Then, the main results can be gotten as follows.

Theorem 2. Starting from any initial values of controlled error system (10), the orbit e $(t)$ converges to the origin ast $\rightarrow \infty$; that $i s$, the synchronization of the stable chaotic financial systems with two different initial conditions is realized by the above controller $U_{1}=\left(k_{1} e_{1}, 0,0\right)^{T}$.

Proof. Differentiating the function $V_{1}$ along the trajectories of the augment system $(*)$, we obtain

$$
\begin{aligned}
\left.\dot{V}_{1}\right|_{(*)}= & e_{1} \dot{e}_{1}+e_{2} \dot{e}_{2}+e_{3} \dot{e}_{3}+\frac{1}{\gamma}\left(k_{1}+L_{1}\right) \dot{k}_{1} \\
= & e_{1}\left(F_{1}(x, e)+k_{1} e_{1}\right)+e_{2} \cdot F_{2}(x, e)+e_{3} \\
& \cdot F_{3}(x, e)+\frac{1}{\gamma}\left(k_{1}+L_{1}\right)\left(-\gamma e_{1}^{2}\right) \\
= & e_{1} \cdot F_{1}(x, e)+e_{2} \cdot F_{2}(x, e)+e_{3} \cdot F_{3}(x, e) \\
& -L_{1} e_{1}^{2} \leq M_{1}\left(e_{1}^{2}+e_{2}^{2}+e_{3}^{2}\right)-L_{1} e_{1}^{2} .
\end{aligned}
$$

Therefore, by (13),

$$
\left.\dot{V}_{1}\right|_{(*)}<0
$$

Then the system is uniformly asymptotically stable at the equilibrium point. So the conclusion is certified.

In order to verify the results of the above theory, we carried out numerical simulations to select the following initial conditions: $x_{0}=(-2,3,4)^{T}, y_{0}=(4,-1,-2)^{T}$, and $k_{1}(0)=$ -1 . In Figure 2, when the controller is activated, the initial errors are $e=\left(e_{1}, e_{2}, e_{3}\right)^{T}=(6,-4,-6)^{T}$ and show the time response of the synchronization errors $e_{1}, e_{2}, e_{3}$ and the error system is dynamic stability. In Figure 3, the state variables are plotted, and $x_{1}, x_{2}, x_{3}$ are synchronized with $y_{1}, y_{2}, y_{3}$. Figures 2 and 3 indicate that the synchronization of the chaotic financial systems is achieved.

\section{Hybrid Synchronization in the Chaotic Financial Systems}

In the above section, we just discuss the synchronization of chaotic financial systems. But in reality, we need to deal with more hybrid synchronization chaotic financial systems. 


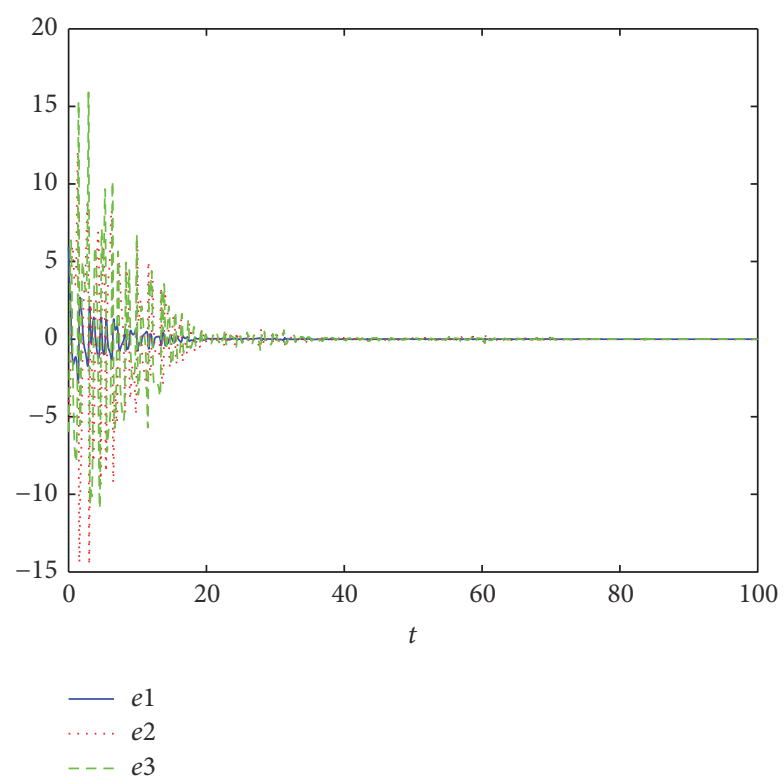

FIGURE 2: Asymptotic stability of error system.
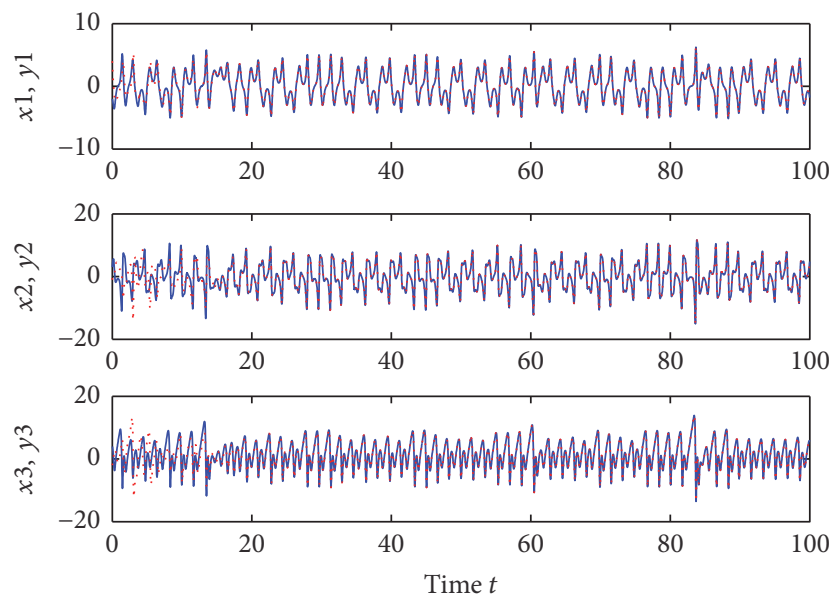

Figure 3: The figure shows that $x_{1}, x_{2}, x_{3}$ are synchronized to $y_{1}, y_{2}, y_{3}$.

Let the antisynchronization error be denoted by $E=x+y$. Therefore, the antisynchronization error dynamic system is given as follows:

$$
\dot{E}=F(y)+F(x) \text {. }
$$

Definition 3. Drive system (2) and response system (4) are called antisynchronization if and only if $\lim _{t \rightarrow \infty}\|E(t)\|=0$.

In [13], the problem of hybrid synchronization in chaotic systems is studied, but no specific discriminant methods and steps are given. In order to determine the synchronization or antisynchronization of the variables in the error system, we give the following algorithm to test the variables that implement the antisynchronization, and the remaining variables are synchronized.

The algorithm is represented as follows.
Step 1. Without loss of generality, first select the variable $x_{1}$. If $F_{1}(x)=F_{11}\left(x_{1}\right)+F_{12}\left(x_{2}, \ldots, x_{n}\right)$ is an odd function or $F_{11}\left(x_{1}\right)=\alpha_{1} x_{1}$, then $E_{1}=x_{1}+y_{1}$ can be made, where $\alpha_{1}$ is a real number.

Step 2. If $F_{12}\left(x_{2}, \ldots, x_{n}\right)=\alpha_{2} x_{2}+F_{13}\left(x_{3}, \ldots, x_{n}\right)$, we should set $E_{2}=x_{2}+y_{2}$, where $\alpha_{2}$ is a real number. Else, if $F_{2}(x)=$ $F_{21}\left(x_{2}\right)+F_{22}\left(x_{1}, x_{3}, \ldots, x_{n}\right)$ is an odd function or $F_{21}\left(x_{2}\right)=$ $\alpha_{3} x_{3}$, we can set $E_{2}=x_{2}+y_{2}$, where $\alpha_{3}$ is a real number. Then, according to the condition that the origin is the equilibrium point of the error system, determine whether $E_{2}=x_{2}+y_{2}$ is suitable. If not, then $e_{2}=y_{2}-x_{2}$.

Step 3. When $i \leq n, E_{i}=y_{i}+x_{i}$ or $e_{i}=y_{i}-x_{i}$ can be set by the similar procedure in Step 2.

According to the above algorithm, because $F_{1}(x)=$ $-m\left(x_{1}+x_{2}\right), F_{1}(x)$ is an odd function, $E_{1}=x_{1}+y_{1}$ can be built; similarly, $E_{2}=x_{2}+y_{2}$ also can be set. If $E_{3}=x_{3}+y_{3}$ was built, then there is

$$
\begin{aligned}
\dot{E}_{3} & =2 n+m\left(x_{1} x_{2}+y_{1} y_{2}\right) \\
& =2 n+m\left(E_{1} E_{2}-x_{1} E_{2}-x_{2} E_{1}+2 x_{1} x_{2}\right) .
\end{aligned}
$$

It is clear that $E=0$ is not an equilibrium point of error system (17), and $F_{3}=n+m x y$ is not an odd function. In fact, the left hand side of error system (17) equals zero. However, the right hand side of error system (18) is not equal to zero but equals $2 n+2 m x_{1} x_{2}$. Therefore, $e_{3}=y_{3}-x_{3}$ can been set and obtain the error system given as follows.

Among them, make $y_{i}=E_{i}-x_{i}(i=1,2), y_{3}=e_{3}+x_{3}$, get the error system

$$
\begin{aligned}
& \dot{E}_{1}=f_{1}\left(x, y, e_{3}, E\right)=-m\left(E_{1}+E_{2}\right) \\
& \dot{E}_{2}=f_{2}\left(x, y, e_{3}, E\right)=-E_{2}-m\left(E_{1} e_{3}+x_{1} e_{3}-x_{3} E_{1}\right) \\
& \dot{e}_{3}=f_{3}\left(x, y, e_{3}, E\right)=m\left(E_{1} E_{2}-x_{1} E_{2}-x_{2} E_{1}\right),
\end{aligned}
$$

where $E=\left(E_{1}, E_{2}\right)^{T}$.

According to the control theory, synchronization error system (18) is represented by the controller $U=\left(u_{1}, u_{2}, u_{3}\right)^{T}$ as follows:

$$
\begin{aligned}
\dot{E}_{1} & =f_{1}\left(x, y, e_{3}, E\right)=-m\left(E_{1}+E_{2}\right)+u_{1} \\
\dot{E}_{2} & =f_{2}\left(x, y, e_{3}, E\right) \\
& =-E_{2}-m\left(E_{1} e_{3}+x_{1} e_{3}-x_{3} E_{1}\right)+u_{2} \\
\dot{e}_{3} & =f_{3}\left(x, y, e_{3}, E\right)=m\left(E_{1} E_{2}-x_{1} E_{2}-x_{2} E_{1}\right)+u_{3} .
\end{aligned}
$$

Equation (18) is a hybrid synchronization error system. Here, similar to the previous discussion, we make the following assumptions about error system (18).

For error system (18), there exists $\lambda_{i}>0(i=1,2,3)$, which is subject to

$$
\begin{aligned}
& E_{i} f_{i}\left(x, y, e_{3}, E\right) \leq \lambda_{i} E_{i}^{2}, \quad i=1,2 \\
& e_{3} f_{3}\left(x, y, e_{3}, E\right) \leq \lambda_{3} e_{3}^{2} .
\end{aligned}
$$


In addition, for uncontrolled synchronization error system (18), if we make $E_{j}=0(j=1,2)$ rather than $e_{3}=0$, then the subsystem $\dot{e}_{3}=F_{3}\left(x, 0, e_{3}, E_{3}\right), \dot{E}_{i}=F_{i}\left(x, 0, e_{3}, E_{i}\right)$ is asymptotically stable, respectively, and $i \neq j(i, j=1,2)$. So we can design a single variable controller $u_{j}=p_{s} E_{j}(s, j=$ $1,2)$, to achieve synchronization and antisynchronization coexistence. Here $\dot{p}_{s}=-\gamma E_{j}^{2}, \gamma$ is a positive numbers and generally $\gamma=1$.

We design some simpler controller for error system, respectively, as follows.

3.1. Control Error System under $U_{2}=\left(p_{1} E_{1}, 0,0\right)^{T}$. If $E_{1}=0$, then the subsystem of (18) is

$$
\begin{aligned}
& \dot{E}_{2}=-E_{2}-m x_{1} e_{3} \\
& \dot{e}_{3}=-m x_{1} E_{2} .
\end{aligned}
$$

It is globally asymptotically stable at the origin.

Now, we add the controller $U_{2}=\left(p_{1} E_{1}, 0,0\right)^{T}$ to response system (4) and the control error system, which is indicated as follows:

$$
\begin{aligned}
& \dot{E}_{1}=-m\left(E_{1}+E_{2}\right)+p_{1} E_{1} \\
& \dot{E}_{2}=-E_{2}-m\left(E_{1} e_{3}+x_{1} e_{3}-x_{3} E_{1}\right) \\
& \dot{e}_{3}=m\left(E_{1} E_{2}-x_{1} E_{2}-x_{2} E_{1}\right),
\end{aligned}
$$

where the feedback gain $p_{1}$ is adapted according to the following update law:

$$
\dot{p}_{1}=-\gamma E_{1}^{2} \text {, }
$$

where $\gamma>0$ is an arbitrary number. In general, we set $\gamma=1$.

In order to facilitate the analysis, make system (22) and (23) be called the augment system $(* *)$, and introduce a Lyapunov function as follows:

$$
V_{2}\left(E, e_{3}, p_{1}\right)=\frac{1}{2}\left(e_{3}^{2}+E^{T} E\right)+\frac{1}{2 \gamma}\left(p_{1}+L_{2}\right)^{2},
$$

where

$$
L_{2}>M_{2} \sup _{x_{1} \neq 0} \frac{e_{3}^{2}+E^{T} E}{E_{1}^{2}}, \quad M_{2}=\max _{1 \leq i \leq 3} \lambda_{i} .
$$

Then, give the following main result.

Theorem 4. Starting from any initial values of controlled error system (22), the orbit $(E(t), e(t))^{T}$ converges to the origin as $t \rightarrow \infty$; that is, the synchronization of the stable chaotic finance systems with two different initial conditions is realized by the above controller $U_{2}=\left(p_{1} E_{1}, 0,0\right)^{T}$.

Proof. Firstly, introduce a Lyapunov function (24), and it satisfied (25).
Then, differentiating the function $V_{2}$ along the trajectories of the augment system, we obtain

$$
\begin{aligned}
\left.\dot{V}_{2}\right|_{(* *)}= & E_{1} \dot{E}_{1}+E_{2} \dot{E}_{2}+e_{3} \dot{e}_{3}+\frac{1}{\gamma}\left(p_{1}+L_{2}\right) \dot{p}_{1} \\
= & E_{1}\left(f_{1}\left(x, y, e_{3}, E\right)+k_{2} E_{1}\right)+E_{2} \\
& \cdot f_{2}\left(x, y, e_{3}, E\right)+e_{3} \cdot f_{3}\left(x, y, e_{3}, E\right) \\
& +\frac{1}{\gamma}\left(k_{2}+L_{2}\right)\left(-\gamma E_{1}^{2}\right) \\
= & E_{1} \cdot f_{1}\left(x, y, E, e_{3}\right)+E_{2} \cdot f_{2}\left(x, y, e_{3}, E\right) \\
& +e_{3} \cdot f_{3}\left(x, y, e_{3}, E\right)-L_{2} E_{1}^{2} \\
\leq & M_{2}\left(E_{1}^{2}+E_{2}^{2}+e_{3}^{2}\right)-L_{2} E_{1}^{2}<0 .
\end{aligned}
$$

By (24) and (25), we derive that

$$
\left.\dot{V}_{2}\right|_{(* *)}<0 .
$$

Then the system is uniformly asymptotically stable at the equilibrium point. So the conclusion is certified.

Remark 5. Theorem 4 shows that the coexistence of synchronization and antisynchronization can be achieved by the above controller $U_{2}=\left(p_{1} E_{1}, 0,0\right)^{T}$ in the same chaotic financial system.

To illustrate the correctness of the above result, we carry out numerical simulations with the same initial conditions and $k_{2}(0)=-1$. In Figure 4 , when the controller is activated, the initial errors are $\left(E_{1}, E_{2}, e_{3}\right)^{T}=(2,2,2)^{T}$ and show the time response of the hybrid synchronization errors, and the error system is dynamic stability. Figure 5 shows that the state variables $x_{1}, x_{2}$ are antisynchronized with $y_{1}, y_{2}$, and $x_{3}$ is synchronized with $y_{3}$. Figures 4 and 5 indicate that the hybrid synchronization of the chaotic financial systems is achieved.

3.2. Control Error System under $U_{3}=\left(0, p_{2} E_{2}, 0\right)^{T}$. If $E_{2}=0$, then subsystem of equation (18) is

$$
\begin{aligned}
& \dot{E}_{1}=-m E_{1} \\
& \dot{e}_{3}=-m x_{2} E_{1} .
\end{aligned}
$$

It is globally asymptotically stable at the origin.

We add the controller $U_{3}=\left(0, p_{2} E_{2}, 0\right)^{T}$ to response system (4) and the control error system, which is indicated as follows:

$$
\begin{aligned}
& \dot{E}_{1}=-m\left(E_{1}+E_{2}\right) \\
& \dot{E}_{2}=-E_{2}-m\left(E_{1} e_{3}+x_{1} e_{3}-x_{3} E_{1}\right)+p_{2} E_{2} \\
& \dot{e}_{3}=m\left(E_{1} E_{2}-x_{1} E_{2}-x_{2} E_{1}\right),
\end{aligned}
$$

where the feedback gain $k_{3}$ is adapted according to the following update law:

$$
\dot{p}_{2}=-\gamma E_{2}^{2},
$$

where $\gamma>0$ is an arbitrary number. 


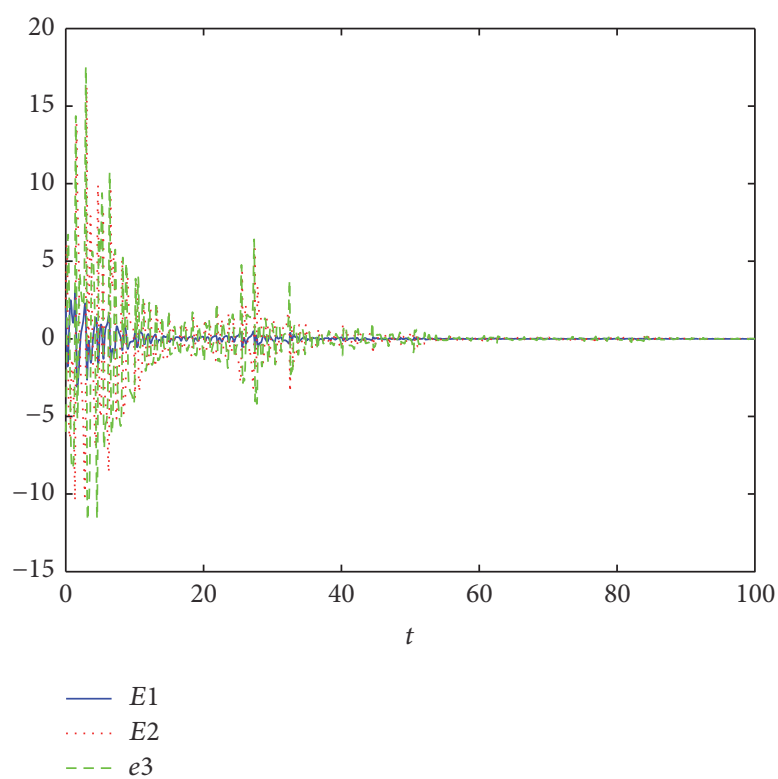

FIGURE 4: Get the error system asymptotically stable with controller $U_{2}=\left(p_{1} E_{1}, 0,0\right)^{T}$.
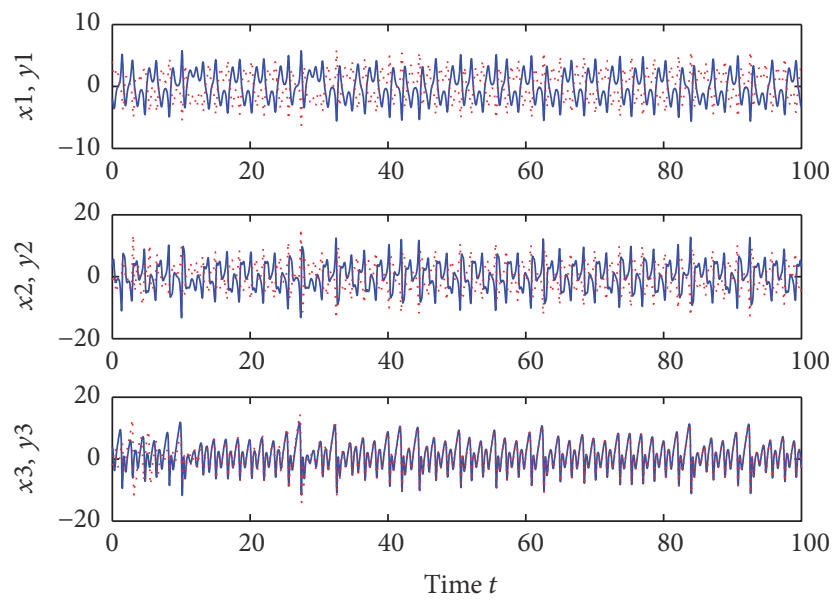

FIGURE 5: This figures shows that $x_{1}, x_{2}$ antisynchronize $y_{1}, y_{2}$, while $x_{3}$ synchronizes $y_{3}$, respectively.

Theorem 6. Starting from any initial values of controlled error system (23), the orbit $(E(t), e(t))^{T}$ converges to the origin as $\rightarrow$ $\infty$; that is, the synchronization of the stable chaotic financial systems with two different initial conditions is realized by the above controller $U_{3}=\left(0, p_{2} E_{2}, 0\right)^{T}$.

The proof is similar to Theorem 4. In fact, we can introduce a Lyapunov function as follows:

$$
V_{3}\left(E, e_{3}, p_{2}\right)=\frac{1}{2}\left(e_{3}^{2}+E^{T} E\right)+\frac{1}{2 \gamma}\left(p_{2}+L_{3}\right)^{2},
$$

where

$$
L_{3}>M_{3} \sup _{x_{2} \neq 0} \frac{e_{3}^{2}+E^{T} E}{E_{2}^{2}}, \quad M_{3}=\max _{1 \leq i \leq 3} \lambda_{i} .
$$

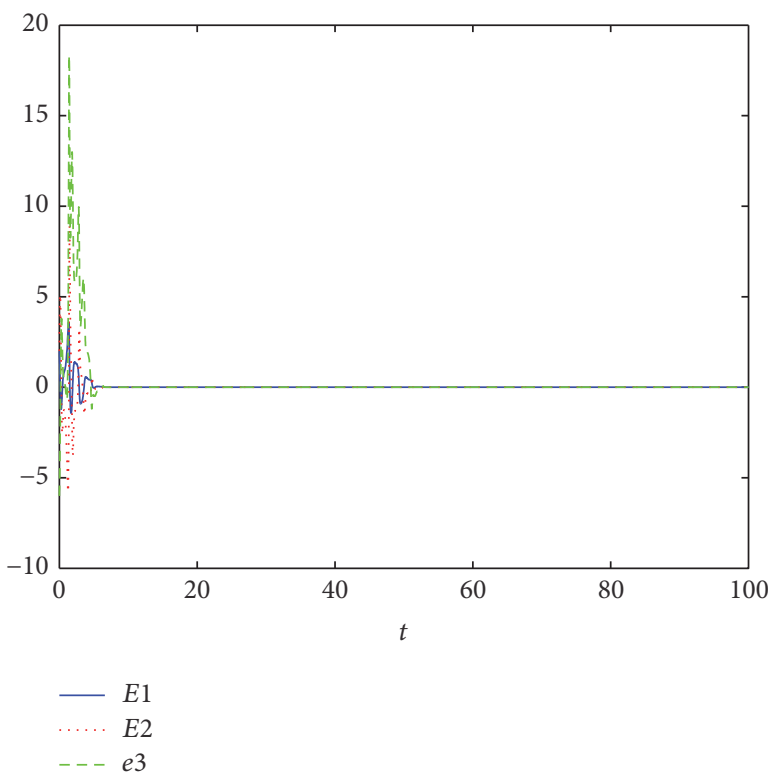

FIGURE 6: Get the error system asymptotically stable with controller $U_{3}=\left(0, p_{2} E_{2}, 0\right)^{T}$.
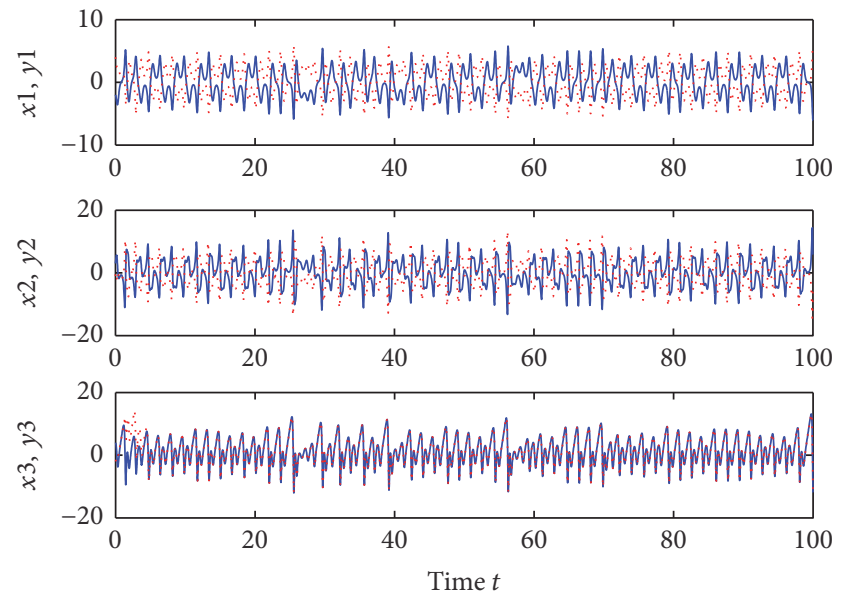

FIGURE 7: The figure shows that $x_{1}, x_{2}$ antisynchronize $y_{1}, y_{2}$, while $x_{3}$ synchronizes $y_{3}$, respectively.

Remark 7. The coexistence of synchronization and antisynchronization can be also achieved by the above controller $U_{3}=\left(0, p_{2} E_{2}, 0\right)^{T}$. Figures 6 and 7 show that the above results.

To illustrate the correctness of the above result, we carry out numerical simulations with the same initial conditions and $k_{2}(0)=-1$. In Figure 6, when the controller is activated, the initial errors are $\left(E_{1}, E_{2}, e_{3}\right)^{T}=(2,2,2)^{T}$ and show the time response of the hybrid synchronization errors and the error system is dynamic stability. Figure 7 shows that the state variables $x_{1}, x_{2}$ are antisynchronized with $y_{1}, y_{2}$ and $x_{3}$ is synchronized with $y_{3}$. Figures 6 and 7 indicate that the hybrid synchronization of the chaotic financial systems is achieved. 
By using different controller for the error of system (18), the hybrid synchronization effects presented by $U_{2}=$ $\left(p_{1} E_{1}, 0,0\right)^{T}$ and $U_{3}=\left(0, p_{2} E_{2}, 0\right)^{T}$ are different. Under the control of $U_{3}=\left(0, p_{2} E_{2}, 0\right)^{T}$, hybrid synchronization effect of the reaction time is short, and under the control of $U_{2}=$ $\left(p_{1} E_{1}, 0,0\right)^{T}$ it is in a long time. Therefore, for the actual financial system, we can design a more appropriate controller according to the time demand of the system to reach the equilibrium state.

\section{Conclusion and Future Work}

In this paper, we have investigated the synchronization and hybrid synchronization of the chaotic financial systems. First, a single variable controller has been presented to synchronize two identical chaotic finance systems with different initial conditions. Second, use a feasible algorithm to determine the partial vector which can realize antisynchronization in chaotic financial system; while other variables can realize synchronization, the hybrid synchronization of the finance system has been realized by a single variable controller as well. It should be pointed out that the obtained controllers in this paper are simpler than those of the existing results. It is convenient to change the controller to get the desired synchronous simulation effect. Numerical simulations have verified the correctness and the effectiveness of the proposed results. There are more complex dynamical behaviors in this finance chaotic system than the normal one. In addition, if the occurrence of time delay or parameter is uncertain, the system is worth further study. It is believed that the system will have broad applications in various chaos-based information systems.

\section{Conflicts of Interest}

The authors declare that there are no conflicts of interest regarding the publication of this article.

\section{Acknowledgments}

This paper was supported in part by Science and Technology Research Project of Chongqing Municipal Education Commission (no. KJ1400428).

\section{References}

[1] E. N. Lorenz, "Deterministic nonperiodic flow," Journal of the Atmospheric Sciences, vol. 20, no. 2, pp. 130-141, 1963.

[2] O. E. Rössler, "An equation for continuous chaos," Physics Letters A, vol. 57, no. 5, pp. 397-398, 1976.

[3] J.-M. Grandmont, "On endogenous competitive business cycles," Econometrica, vol. 53, no. 5, pp. 995-1045, 1985.

[4] E. Ott, C. Grebogi, and J. A. Yorke, "Controlling chaos," Physical Review Letters, vol. 64, no. 11, pp. 1196-1199, 1990.

[5] D. Huang and H. Li, Theory and Method of Nonlinear Economics, Sichuan University Press, Chengdu, China, 1993.

[6] J. H. Ma, B. Ren, and Y. S. Chen, "Impulsive control of chaotic attractors in nonlinear chaotic systems," Applied Mathematics and Mechanics, vol. 25, no. 9, pp. 971-976, 2004.
[7] L. M. Pecora and T. L. Carroll, "Synchronization in chaotic systems," Physical Review Letters, vol. 64, no. 8, pp. 821-824, 1990.

[8] Y. Xu, C. Xie, Y. Wang, W. Zhou, and J. Fang, "Chaos projective synchronization of the chaotic finance system with parameter switching perturbation and input time-varying delay," Mathematical Methods in the Applied Sciences, vol. 38, no. 17, pp. 42794288, 2015.

[9] J.Zheng and B. Du, "Projective synchronization of hyperchaotic financial systems," Discrete Dynamics in Nature and Society, vol. 2015, Article ID 782630, 9 pages, 2015.

[10] J. Ding, W. Yang, and H. Yao, "A new modified hyperchaotic finance system and its control," International Journal of Nonlinear Science, vol. 8, no. 1, pp. 59-66, 2009.

[11] G. Yang, "A single parameter linear feedback control of a new chaotic system," Journal of Yulin College, vol. 19, no. 4, pp. 2022, 2009 (Chinese).

[12] S. Hammami, M. Benrejeb, M. Feki, and P. Borne, "Feedback control design for Rössler and Chen chaotic systems antisynchronization," Physics Letters A, vol. 374, no. 28, pp. 28352840,2010

[13] W. Li and Q. Li, "Dynamic analysis and synchronization and anti-synchronization of a new chaotic system," Application Research of Computers, vol. 32, no. 9, pp. 2659-2663, 2015 (Chinese).

[14] S. Zhang, "A synchronization and anti-synchronization of a new hyperchaotic system," Journal of Changshu Institute of Technology, vol. 10, pp. 32-36, 2012 (Chinese).

[15] L. Ren and R. Guo, "Synchronization and antisynchronization for a class of chaotic systems by a simple adaptive controller," Mathematical Problems in Engineering, vol. 2015, Article ID 434651, 7 pages, 2015.

[16] M. Hu, Z. Xu, and R. Zhang, "Full state hybrid projective synchronization in continuous-time chaotic (hyperchaotic) systems," Communications in Nonlinear Science and Numerical Simulation, vol. 13, no. 2, pp. 456-464, 2008.

[17] R. Guo, B. Li, and Z. Yan, "Serval control problems of a novel finance chaotic system," Chinese Control and Decision Conference, no. 27, pp. 3864-3869, 2015.

[18] L. Ren, R. Guo, and U. E. Vincent, "Coexistence of synchronization and anti-synchronization in chaotic systems," Archives of Control Sciences, vol. 26(62), no. 1, pp. 69-79, 2016.

[19] R. Zhang, "Bifurcation analysis for a kind of nonlinear finance system with delayed feedback and its application to control of chaos," Journal of Applied Mathematics, vol. 2012, Article ID 316390, 18 pages, 2012. 


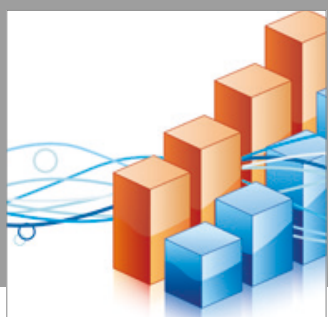

Advances in

Operations Research

vatersals

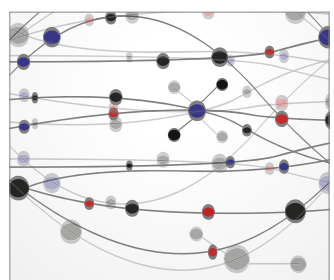

\section{The Scientific} World Journal
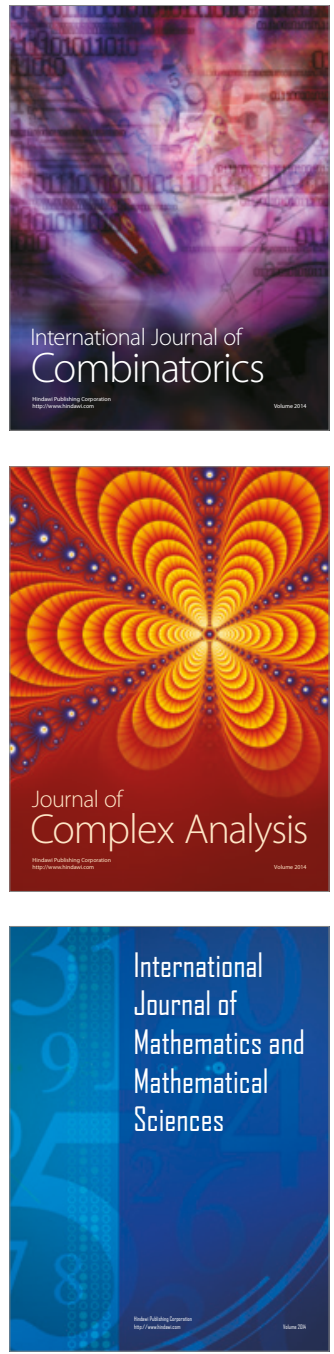
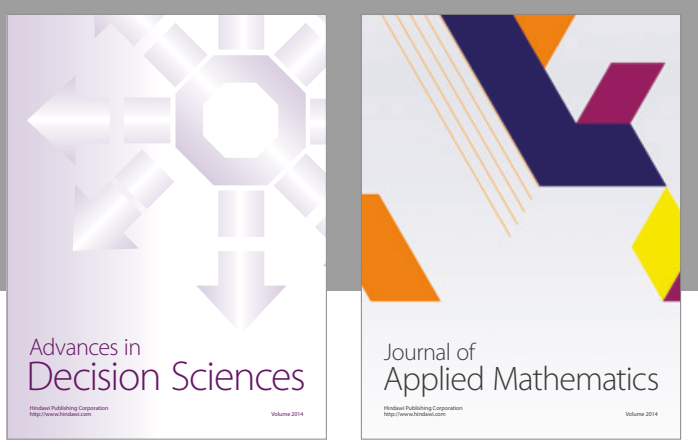

Algebra

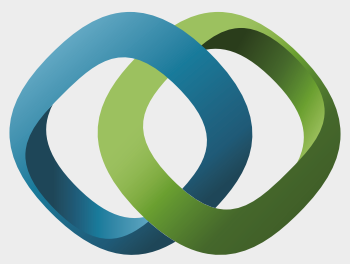

\section{Hindawi}

Submit your manuscripts at

https://www.hindawi.com
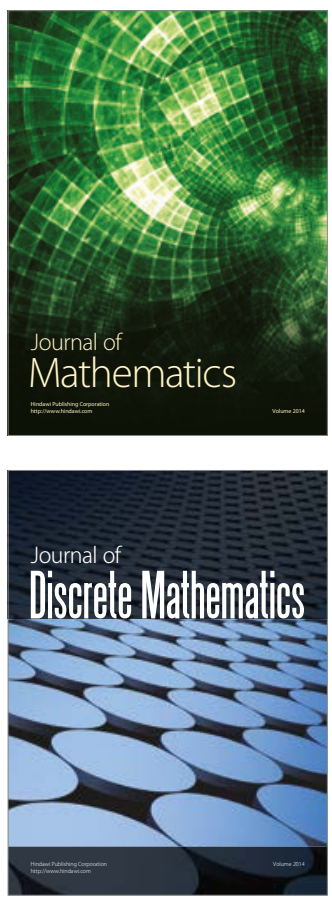

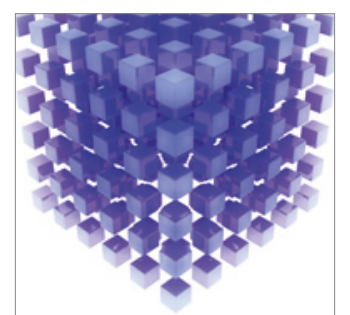

Mathematical Problems in Engineering
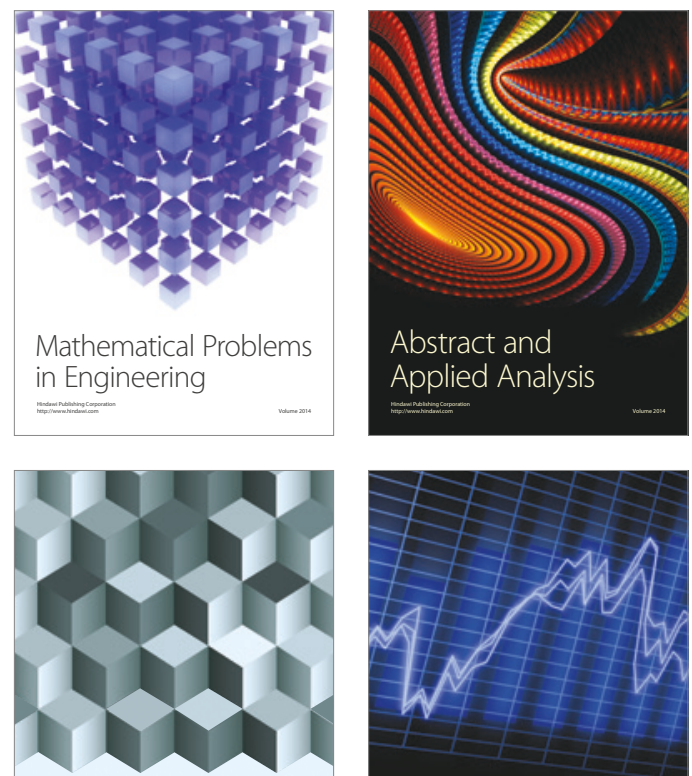

Journal of

Function Spaces

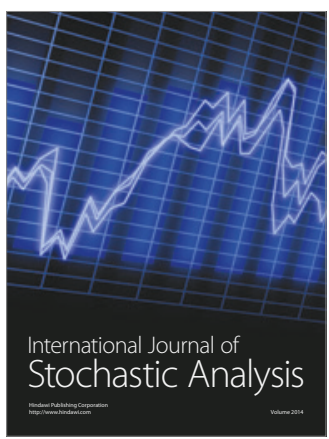

Probability and Statistics
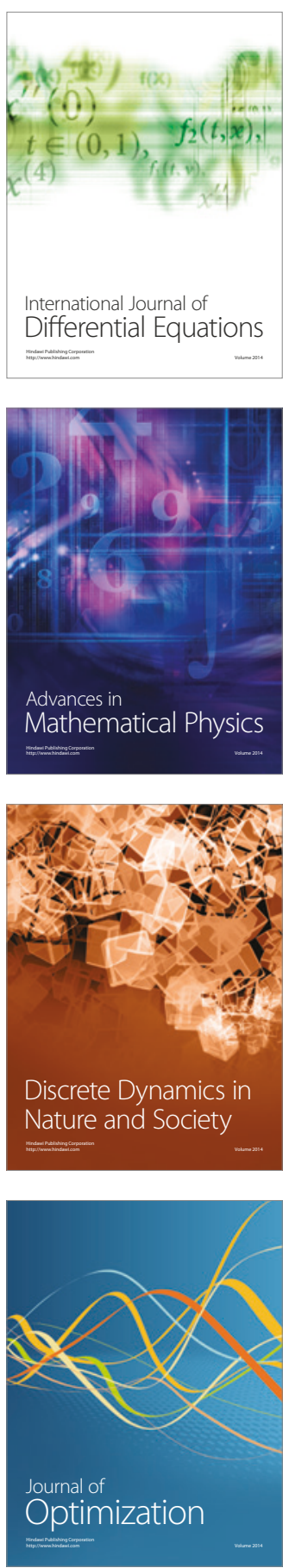